ANNALES

POLONICI MATHEMATICI

LXXVIII.2 (2002)

\title{
Estimates of capacity of self-similar measures
}

\author{
by JózeF MyjaK (L’Aquila and Kraków) and \\ Tomasz Szarek (Katowice and Rzeszów)
}

\begin{abstract}
We give lower and upper estimates of the capacity of self-similar measures generated by iterated function systems $\left\{\left(S_{i}, p_{i}\right): i=1, \ldots, N\right\}$ where $S_{i}$ are bilipschitzean transformations.
\end{abstract}

1. Introduction. The idea of dimension of measures is fundamental in measure theory and it also occurs in diverse branches of mathematics. For example, it is a basic tool in the study of attractors of dynamical systems, in particular in the study of attractors (also called fractals) generated by iterated function systems, or more generally, fractals generated by Markov chains (see $[1-6,15,16,18,23]$ ). Various notions of dimension have been proposed: Hausdorff dimension, box dimension, entropy dimension, correlation dimension. These concepts were widely investigated and used. Closely related to Hausdorff dimension is capacity, introduced by Kolmogorov (see [14]). This capacity, however, does not distinguish between a set and its closure. Ledrappier [17] has made some modification to correct this insensitivity. While the other concepts mentioned here have been extensively studied, Ledrappier's version of capacity does not seem to be sufficiently explored. In this paper we give lower and upper estimates of Ledrappier's capacity of measures invariant with respect to iterated function systems of functions which are bi-lipschitzean.

The calculation of dimensions has been performed by several authors inspired by Hutchinson's elegant treatment [13]. For an account of the technique involved, generalizations and improvements see $[2-4,12,18,20]$. Our approach is also based on this idea.

2. Notations and preliminaries. Throughout this paper $(X, \varrho)$ denotes a Polish space and this assumption will not be repeated in the state-

2000 Mathematics Subject Classification: Primary 47A35; Secondary 28D15.

Key words and phrases: Markov operator, iterated function system, invariant measure, capacity of measure. 
ment of theorems. By $B(x, r)$ we denote the closed ball in $X$ with center at $x$ and radius $r$. For $A \subset X, A \neq \emptyset$, we denote by $\operatorname{diam} A$ the diameter of $A$ and by $1_{A}$ the characteristic function of $A$. Moreover, for $A, B \subset X$, $A, B \neq \emptyset$, we define

$$
\operatorname{dist}(A, B)=\inf \{\varrho(x, y): x \in A, y \in B\} .
$$

As usual, $\mathbb{R}$ stands for the set of all reals and $\mathbb{N}$ for the set of all positive integers. Moreover set $\mathbb{R}_{+}=[0, \infty)$.

We denote by $\mathcal{B}(X)$ the $\sigma$-algebra of Borel subsets of $X$ and by $\mathcal{M}$ the family of all finite Borel measures on $X$. Moreover, $\mathcal{M}_{1}$ denotes the family of all $\mu \in \mathcal{M}$ such that $\mu(X)=1$, and $\mathcal{M}_{\mathrm{s}}=\left\{\mu_{1}-\mu_{2}: \mu_{1}, \mu_{2} \in \mathcal{M}\right\}$ is the space of all finite signed measures.

Finally, $B(X)$ stands for the space of all bounded Borel measurable functions $f: X \rightarrow \mathbb{R}$ and $C(X)$ for the subspace of $B(X)$ of all bounded continuous functions. In both spaces the norm is given by the formula

$$
\|f\|_{0}=\sup _{x \in X}|f(x)| .
$$

For $f \in B(X)$ and $\nu \in \mathcal{M}_{\mathrm{s}}$ we write

$$
\langle f, \nu\rangle=\int_{X} f(x) \nu(d x) .
$$

We say that a sequence $\left(\mu_{n}\right)_{n \geq 1} \subset \mathcal{M}$ converges weakly to a measure $\mu \in \mathcal{M}$ if $\lim _{n \rightarrow \infty}\left\langle f, \mu_{n}\right\rangle=\langle f, \mu\rangle$ for every $f \in C(X)$.

We endow $\mathcal{M}_{\mathrm{s}}$ with the Fortet-Mourier norm (see [11]) given by

$$
\|\nu\|=\sup \{|\langle f, \nu\rangle|: f \in F\},
$$

where $F$ is the set of all functions $f \in C(X)$ such that $\|f\|_{0} \leq 1$ and $|f(x)-f(y)| \leq \varrho(x, y)$ for $x, y \in X$. It is known that the convergence

$$
\lim _{n \rightarrow \infty}\left\|\mu_{n}-\mu\right\|=0 \quad \text { for } \mu_{n}, \mu \in \mathcal{M}_{1}
$$

is equivalent to the weak convergence of the sequence $\left(\mu_{n}\right)_{n \geq 1}$ to $\mu$ (see [7]).

An operator $P: \mathcal{M} \rightarrow \mathcal{M}$ is called a Markov operator if

$$
P\left(\lambda_{1} \mu_{1}+\lambda_{2} \mu_{2}\right)=\lambda_{1} P \mu_{1}+\lambda_{2} P \mu_{2} \quad \text { for } \lambda_{1}, \lambda_{2} \in \mathbb{R}_{+} \text {and } \mu_{1}, \mu_{2} \in \mathcal{M}
$$

and

$$
P \mu(X)=\mu(X) \quad \text { for } \mu \in \mathcal{M} .
$$

A measure $\mu$ is called stationary (or invariant) with respect to the operator $P$ if $P \mu=\mu$. A Markov operator $P$ is called asymptotically stable if there exists an invariant probability measure $\mu_{*}$ such that

$$
\lim _{n \rightarrow \infty}\left\|P^{n} \mu-\mu_{*}\right\|=0 \quad \text { for } \mu \in \mathcal{M}_{1} .
$$

Clearly, the stationary measure is unique if $P$ is asymptotically stable. 
Let $\mu \in \mathcal{M}_{1}$. For given $\varepsilon>0$ and $C \subset X$ we denote by $N_{C}(\varepsilon)$ the minimal number of $\varepsilon$-balls needed to cover the set $C$. Further, for $\varepsilon, \eta>0$ we define

$$
N(\varepsilon, \eta)=\inf \left\{N_{C}(\varepsilon): C \subset X \text { and } \mu(C)>1-\eta\right\} .
$$

Then the quantities

$$
\operatorname{Cap}_{L}(\mu)=\sup _{\eta>0} \liminf _{\varepsilon \rightarrow 0} \frac{\log N(\varepsilon, \eta)}{-\log \varepsilon}
$$

and

$$
\overline{\operatorname{Cap}}_{L}(\mu)=\sup _{\eta>0} \limsup _{\varepsilon \rightarrow 0} \frac{\log N(\varepsilon, \eta)}{-\log \varepsilon}
$$

are called the lower and upper capacity of $\mu$, respectively.

The above definitions were introduced by Ledrappier (see [17, 22, 25]) and are closely related to Kolmogorov dimensions.

REMARK 2.1. In the definitions of the lower and upper capacity we can replace the continuous variable $\varepsilon$ by a decreasing sequence $\left(\varepsilon_{n}\right)_{n \geq 1}$ with $\log \varepsilon_{n+1} / \log \varepsilon_{n} \rightarrow 1$.

Assume now we are given a sequence of continuous transformations

$$
S_{i}: X \rightarrow X \quad \text { for } i=1, \ldots, N
$$

and a probability vector

$$
p_{i}: X \rightarrow[0,1] \quad \text { for } i=1, \ldots, N,
$$

where the $p_{i}$ are continuous functions satisfying

$$
p_{i}(x)>0 \quad \text { and } \quad \sum_{i=1}^{N} p_{i}(x)=1 \quad \text { for } x \in X .
$$

Such a system is denoted by $(S, p)_{N}$ and called an iterated function system (briefly IFS).

Having an IFS $(S, p)_{N}$ we define the corresponding Markov operator $P: \mathcal{M} \rightarrow \mathcal{M}$ by

$$
P \mu(A)=\sum_{i=1}^{N} \int_{S_{i}^{-1}(A)} p_{i}(x) \mu(d x) \quad \text { for } A \in \mathcal{B}(X)
$$

and its dual operator $U: B(X) \rightarrow B(X)$ by

$$
U f(x)=\sum_{i=1}^{N} p_{i}(x) f\left(S_{i}(x)\right) .
$$


We say that an IFS $(S, p)_{N}$ is asymptotically stable if the corresponding Markov operator $P$ is asymptotically stable. A measure $\mu_{*} \in \mathcal{M}$ is called $i n$ variant for the IFS $(S, p)_{N}$ if it is invariant with respect to the corresponding Markov operator $P$.

We assume that $S_{i}: X \rightarrow X, i=1, \ldots, N$, are bi-lipschitzean transformations, i.e. there exist constants $l_{i}, L_{i}>0$ such that

$$
l_{i} \varrho(x, y) \leq \varrho\left(S_{i}(x), S_{i}(y)\right) \leq L_{i} \varrho(x, y) \quad \text { for } x, y \in X .
$$

Throughout this paper $l_{1}, \ldots, l_{N}$ and $L_{1}, \ldots, L_{N}$ always stand for the constants satisfying (2.3). Moreover, we assume that

$$
\begin{gathered}
\Gamma_{0}=\sup _{x \in X} \prod_{i=1}^{N} L_{i}^{p_{i}(x)}<1, \\
\alpha_{0}=\min _{1 \leq i \leq N} \inf _{x \in X} p_{i}(x)>0, \\
\sum_{i=1}^{N}\left|p_{i}(x)-p_{i}(y)\right| \leq \omega(\varrho(x, y)) \quad \text { for } x, y \in X,
\end{gathered}
$$

where $\omega: \mathbb{R}_{+} \rightarrow \mathbb{R}_{+}$is a nondecreasing concave function satisfying the Dini condition

$$
\int_{0}^{\eta} \frac{\omega(t)}{t} d t<\infty \quad \text { for some } \eta>0 .
$$

The following constants will play a crucial role:

$$
\begin{aligned}
\Delta_{0} & =\sup _{x \in X} \prod_{i=1}^{N} p_{i}(x)^{p_{i}(x)}, \\
\delta_{0} & =\inf _{x \in X} \prod_{i=1}^{N} p_{i}(x)^{p_{i}(x)}, \\
\gamma_{0} & =\inf _{x \in X} \prod_{i=1}^{N} l_{i}^{p_{i}(x)} .
\end{aligned}
$$

Obviously $\Delta_{0}, \delta_{0}, \gamma_{0} \in(0,1)$.

We say that a family of transformations $S_{1}, \ldots, S_{N}$ satisfies the strong Moran condition (see [19]) if there exists a bounded closed subset $F$ of $X$ and a constant $\sigma>0$ such that

$$
\begin{gathered}
\bigcup_{i=1}^{N} S_{i}(F) \subset F, \\
\operatorname{dist}\left(S_{i}(F), S_{j}(F)\right) \geq \sigma \quad \text { for } i \neq j .
\end{gathered}
$$


Proposition 2.1. If an IFS $(S, p)_{N}$ satisfies conditions (2.3)-(2.7), then it is asymptotically stable.

Proof. See [23].

Let $\Omega=\{1, \ldots, N\}^{\infty}=\left\{\left(i_{1}, i_{2}, \ldots\right): i_{k} \in\{1, \ldots, N\}\right.$ for every $\left.k \in \mathbb{N}\right\}$ and $\Omega_{*}=\bigcup_{n=1}^{\infty} \Omega_{n}$, where $\Omega_{n}=\{1, \ldots, N\}^{n}$. Observe that $\Omega_{*}$ (resp. $\Omega$ ) is the space of all finite (resp. infinite) sequences of elements $i_{k} \in$ $\{1, \ldots, N\}$. For $k \in \mathbb{N}$ we set $\Omega_{\leq k}=\bigcup_{n=1}^{k} \Omega_{n}$ and $\Omega_{\geq k}=\bigcup_{n=k}^{\infty} \Omega_{n}$. For $\mathbf{i}=\left(i_{1}, \ldots, i_{n}\right) \in \Omega_{*}$ let $|\mathbf{i}|=n$ denote the length of $\mathbf{i}$. If $\mathbf{i} \in \Omega$ we assume that $|\mathbf{i}|=\infty$. For $\mathbf{i} \in \Omega \cup \Omega_{*}$ and $m \in \mathbb{N}, m \leq|\mathbf{i}|$, we set $\mathbf{i} \mid m=\left(i_{1}, \ldots, i_{m}\right)$. We say that $\mathbf{i}<\mathbf{j}$ with $\mathbf{i} \in \Omega_{*}$ and $\mathbf{j} \in \Omega \cup \Omega_{*}$ if $|\mathbf{j}|>n$ and $\mathbf{j} \mid n=\mathbf{i}$, where $n=|\mathbf{i}|$. Finally, for $\mathbf{i}=\left(i_{1}, \ldots, i_{n}\right) \in \Omega_{*}$, we write $\mathbf{i}^{-1}=\left(i_{n}, \ldots, i_{1}\right)$.

A subset $\Lambda \subset \Omega$ is called a cylinder if there exists $\mathbf{i}=\left(i_{1}, \ldots, i_{n}\right) \in \Omega_{*}$ such that

$$
\Lambda=\Lambda(\mathbf{i})=\{\mathbf{j} \in \Omega: \mathbf{j} \mid n=\mathbf{i}\} .
$$

We denote by $\mathcal{A}$ the $\sigma$-algebra of subsets of $\Omega$ which is generated by such cylinders.

Given an IFS $(S, p)_{N}$ and a point $x \in X$ we denote by $\mathbb{P}_{x}$ the probability measure on $\mathcal{A}$ defined on the cylinder $\Lambda(\mathbf{i}), \mathbf{i}=\left(i_{1}, \ldots, i_{n}\right) \in \Omega_{*}$, by the formula

$$
\mathbb{P}_{x}(\Lambda(\mathbf{i}))=p_{i_{1}}(x) p_{i_{2}}\left(S_{i_{1}}(x)\right) \ldots p_{i_{n}}\left(S_{i_{n-1}} \circ \ldots \circ S_{i_{1}}(x)\right) .
$$

It is clear that the above formula defines the unique probability measure for realization of the Markov process starting from the point $x$ for the given $\operatorname{IFS}(S, p)_{N}($ see $[2])$.

For convenience, in what follows we write $\mathbb{P}_{x}(\mathbf{i})$ instead of $\mathbb{P}_{x}(\Lambda(\mathbf{i}))$ and $\mathbb{P}_{x}(A)$ instead of $\mathbb{P}_{x}(\Lambda(A))$, where $A \subset \Omega_{*}$ and $\Lambda(A)=\bigcup_{\mathbf{i} \in A} \Lambda(\mathbf{i})$. Moreover, for $\mathbf{i} \in \Omega_{n}$ we write

$$
S_{\mathbf{i}}=S_{i_{n}} \circ \ldots \circ S_{i_{1}}
$$

Proposition 2.2. For every $\mathbf{i}=\left(i_{1}, \ldots, i_{n}\right) \in \Omega_{*}$, we have

$$
\begin{gathered}
\mathbb{P}_{x}(\mathbf{i})=p_{i_{1}}(x) \mathbb{P}_{S_{i_{1}}(x)}\left(\left(i_{2}, \ldots, i_{n}\right)\right), \\
\mathbb{P}_{x}(\mathbf{i})=p_{i_{n}}\left(S_{i_{n-1}} \circ \ldots \circ S_{1}(x)\right) \mathbb{P}_{x}\left(\left(i_{1}, \ldots, i_{n-1}\right)\right), \\
\sum_{i=1}^{N} \mathbb{P}_{x}((\mathbf{i}, i))=\mathbb{P}_{x}(\mathbf{i}), \\
\mathbb{P}_{x}(\mathbf{i} \mid k) \geq \mathbb{P}_{x}(\mathbf{i} \mid m) \quad \text { if } k \leq m \leq n .
\end{gathered}
$$

Proof. Follows immediately from the definition of $\mathbb{P}_{x}$.

3. Auxiliary results. Throughout this section we assume that an IFS $(S, p)_{N}$ is given and $\mathbb{P}_{x}$ is the corresponding probability measure on $\Omega$ given by (2.13). Using a standard martingale argument we prove the following 
Lemma 3.1. Assume that an IFS $(S, p)_{N}$ satisfies conditions (2.3)-(2.7). Let $f_{i}: X \rightarrow \mathbb{R}_{+}, i=1, \ldots, N$, be bounded continuous functions such that

$$
\min _{1 \leq i \leq N} \inf _{x \in X} f_{i}(x)>0 .
$$

Then for every $x \in X$ there exists a measurable set $\Omega_{x} \subset \Omega$ with $\mathbb{P}_{x}\left(\Omega_{x}\right)=1$ such that, for all $\left(i_{1}, i_{2}, \ldots\right) \in \Omega_{x}$,

(3.1) $\quad \limsup _{n \rightarrow \infty} \frac{1}{n} \log \left(f_{i_{1}}(x) f_{i_{2}}\left(S_{i_{1}}(x)\right) \ldots f_{i_{n}}\left(S_{i_{n-1}} \circ \ldots \circ S_{i_{1}}(x)\right)\right) \leq \log \Delta$,

$$
\liminf _{n \rightarrow \infty} \frac{1}{n} \log \left(f_{i_{1}}(x) f_{i_{2}}\left(S_{i_{1}}(x)\right) \ldots f_{i_{n}}\left(S_{i_{n-1}} \circ \ldots \circ S_{i_{1}}(x)\right)\right) \geq \log \delta
$$

where

$$
\begin{aligned}
& \Delta=\sup _{x \in X} \prod_{i=1}^{N} f_{i}(x)^{p_{i}(x)}, \\
& \delta=\inf _{x \in X} \prod_{i=1}^{N} f_{i}(x)^{p_{i}(x)} .
\end{aligned}
$$

Proof. To prove (3.1), fix $x \in X$ and for each $n \in \mathbb{N}$ define $X_{n}: \Omega \rightarrow \mathbb{R}$ by

$$
X_{n}(\mathbf{i})=\log \left(f_{i_{n}}\left(S_{i_{n-1}} \circ \ldots \circ S_{i_{1}}(x)\right)\right) .
$$

For $\mathbf{i}=\left(i_{1}, \ldots, i_{n}\right) \in \Omega_{*}$, we denote by $\mathcal{A}(\mathbf{i})$ the $\sigma$-algebra generated by the cylinders $\left\{\Lambda(\mathbf{j}): \mathbf{j} \in \Omega_{*}, \mathbf{j} \geq \mathbf{i}\right\}$. Moreover, let $\mathbb{E}_{x}$ denote the expectation with respect to the probability measure $\mathbb{P}_{x}$ on $\Omega$.

Fix $\mathbf{i}=\left(i_{1}, i_{2}, \ldots\right) \in \Omega$. We have

$$
\mathbb{E}_{x}\left(X_{n} \mid \mathcal{A}\left(i_{1}, \ldots, i_{n-1}\right)\right)=\sum_{i=1}^{N} p_{i}\left(S_{i_{n-1}} \circ \ldots \circ S_{i_{1}}(x)\right) X_{n}\left(\left(i_{1}, \ldots, i_{n-1}, i\right)\right) .
$$

By (3.3) we have

$$
\sum_{i=1}^{N} p_{i}\left(S_{i_{n-1}} \circ \ldots \circ S_{i_{1}}(x)\right) \log \left(f_{i}\left(S_{i_{n-1}} \circ \ldots \circ S_{i_{1}}(x)\right)\right) \leq \log \Delta .
$$

Now let $Y_{n}=X_{n}-\mathbb{E}_{x}\left(X_{n} \mid \mathcal{A}\left(i_{1}, \ldots, i_{n-1}\right)\right)$. Then

$$
\left|Y_{n}(\mathbf{i})\right| \leq 2 \sup _{\mathbf{i} \in \Omega}\left|X_{n}(\mathbf{i})\right| \quad \mathbb{P}_{x^{-}} \text {a.s. }
$$

Write

$$
M=2 \sup _{\mathbf{i} \in \Omega}\left|X_{n}(\mathbf{i})\right|<\infty .
$$

Define

$$
Z_{n}=\sum_{k=1}^{n} \frac{Y_{k}}{k} \quad \text { for } n \in \mathbb{N}
$$


It is easy to see that $\left(Z_{n}\right)_{n \geq 1}$ is a martingale. Since $Y_{k}$ and $Y_{l}$ for $k \neq l$ are mutually orthogonal, we have

$$
\mathbb{E}_{x}\left(Z_{n}^{2}\right) \leq M^{2} \sum_{k=1}^{\infty} \frac{1}{k^{2}} .
$$

Hence $\left(Z_{n}\right)_{n \geq 1}$ is an $\mathbb{L}^{2}$-bounded martingale, and so $\left(Z_{n}\right)_{n \geq 1}$ is convergent a.s. Then by Kronecker's lemma (see [7])

$$
\lim _{n \rightarrow \infty} \frac{1}{n} \sum_{k=1}^{n} Y_{k}=0 \quad \mathbb{P}_{x^{-}} \text {a.s. }
$$

Thus

$$
\limsup _{n \rightarrow \infty} \frac{1}{n} \sum_{k=1}^{\infty} X_{k}-\log \Delta \leq 0 \quad \mathbb{P}_{x} \text {-a.s. }
$$

whence (3.1) follows immediately.

Replacing $f_{i}$ with $1 / f_{i}$ and using the same argument gives (3.2).

A finite set $\mathcal{L} \subset \Omega_{*}$ is called fundamental for the $\operatorname{IFS}(S, p)_{N}$ if

$$
\sum_{\mathbf{i} \in \mathcal{L}} \mathbb{P}_{x}(\mathbf{i})=1 \quad \text { for every } x \in X
$$

and there are no $\mathbf{i}, \mathbf{j} \in \mathcal{L}$ such that $\mathbf{i}<\mathbf{j}$. Set

$$
|\mathcal{L}|=\max \{|\mathbf{i}|: \mathbf{i} \in \mathcal{L}\} .
$$

Lemma 3.2. Let $\mathcal{L} \subset \Omega_{*}$ be a fundamental set for the $\operatorname{IFS}(S, p)_{N}$. If $\mathbf{i}=\left(i_{1}, \ldots, i_{n}\right) \in \mathcal{L}$ and $n=|\mathcal{L}|$, then $\left(i_{1}, \ldots, i_{n-1}, i\right) \in \mathcal{L}$ for every $i \in$ $\{1, \ldots, N\}$.

Proof. First observe that $\Lambda(\mathbf{i}) \cap \Lambda(\mathbf{j})=\emptyset$ for every $\mathbf{i}, \mathbf{j} \in \mathcal{L}, \mathbf{i} \neq \mathbf{j}$. Now, suppose for a contradiction that there is $\mathbf{i}=\left(i_{1}, \ldots, i_{n}\right) \in \mathcal{L}$ such that $\left(i_{1}, \ldots, i_{n-1}, i\right) \notin \mathcal{L}$ for some $i \in\{1, \ldots, N\}$. It is easy to verify that $\Lambda\left(i_{1}, \ldots, i_{n-1}, i\right) \cap \Lambda(\mathbf{j})=\emptyset$ for every $\mathbf{j} \in \mathcal{L}$. Since $\mathbb{P}_{x}$ is a probability measure and $\mathbb{P}_{x}(\mathbf{i})>0$ for every $\mathbf{i} \in \Omega_{*}$, we have

$$
\sum_{\mathbf{i} \in \mathcal{L}} \mathbb{P}_{x}(\mathbf{i}) \leq 1-\mathbb{P}_{x}\left(\left(i_{1}, \ldots, i_{n-1}, i\right)\right)<1,
$$

which contradicts (3.5).

Remark 3.1. Note that for every $n \in \mathbb{N}$ there exists a fundamental set $\mathcal{L}$ for $(S, p)_{N}$ such that $\mathcal{L} \subset \Omega_{\leq n}$.

Lemma 3.3. Assume that an IFS $(S, p)_{N}$ satisfies conditions (2.3)-(2.7). Let $\mu_{*} \in \mathcal{M}_{1}$ be its unique invariant measure. Then for every fundamental 
set $\mathcal{L} \subset \Omega_{*}$ we have

$$
\mu_{*}(A)=\sum_{\mathbf{i} \in \mathcal{L}} \int_{X} \mathbb{P}_{x}\left(\mathbf{i}^{-1}\right) 1_{A}\left(S_{\mathbf{i}^{-1}}(x)\right) \mu_{*}(d x) \quad \text { for } A \in \mathcal{B}(X) .
$$

Proof. We use induction on $n$, where $\mathcal{L} \subset \Omega_{\leq n}$.

Suppose first that $\mathcal{L} \subset \Omega_{1}$. Since $p_{i}(x)>0$ for $x \in X$ and $i=1, \ldots, N$, it follows immediately that $\mathcal{L}=\{1, \ldots, N\}$ and (3.6) is obviously satisfied.

Now suppose that (3.6) holds for every $\mathcal{L} \subset \Omega_{\leq n}$, and take $\mathcal{L} \subset \Omega_{\leq n+1}$. Using the invariance of $\mu_{*}$ and (2.2), for $f \in B(X)$ we have

$$
\begin{aligned}
\int_{X} f(x) \mu_{*}(d x) & =\int_{X} f(x) P \mu_{*}(d x)=\int_{X} U f(x) \mu_{*}(d x) \\
& =\sum_{i=1}^{N} \int_{X} p_{i}(x) f\left(S_{i}(x)\right) \mu_{*}(d x) .
\end{aligned}
$$

Set

$$
\mathcal{L}_{n+1}=\{\mathbf{i} \in \mathcal{L}:|\mathbf{i}|=n+1\}, \quad \mathcal{L}_{n+1}^{n}=\left\{\mathbf{i} \mid n: \mathbf{i} \in \mathcal{L}_{n+1}\right\} .
$$

We assume that $\mathcal{L}_{n+1} \neq \emptyset$ (otherwise there is nothing to prove). Let $A \in \mathcal{B}(X)$. Using in succession Lemma 3.2, formula (2.14) and (3.7) we have

$$
\begin{aligned}
\sum_{\mathbf{i} \in \mathcal{L}_{n+1}} \int_{X} \mathbb{P}_{x}\left(\mathbf{i}^{-1}\right) 1_{A}\left(S_{\mathbf{i}^{-1}}(x)\right) \mu_{*}(d x) & \\
= & \sum_{\mathbf{j} \in \mathcal{L}_{n+1}^{n}} \sum_{i=1}^{N} \int_{X} \mathbb{P}_{x}\left((\mathbf{j}, i)^{-1}\right) 1_{A}\left(S_{(\mathbf{j}, i)^{-1}}(x)\right) \mu_{*}(d x) \\
& =\sum_{\mathbf{j} \in \mathcal{L}_{n+1}^{n}} \sum_{i=1}^{N} \int_{X} p_{i}(x) \mathbb{P}_{S_{i}(x)}\left(\mathbf{j}^{-1}\right) 1_{A}\left(S_{\mathbf{j}^{-1}}\left(S_{i}(x)\right)\right) \mu_{*}(d x) \\
& =\sum_{\mathbf{j} \in \mathcal{L}_{n+1}^{n}} \int_{X} \mathbb{P}_{x}\left(\mathbf{j}^{-1}\right) \mathbf{1}_{A}\left(S_{\mathbf{j}^{-1}}(x)\right) \mu_{*}(d x) .
\end{aligned}
$$

Now setting $\mathcal{L}^{*}=\left(\mathcal{L} \backslash \mathcal{L}_{n+1}\right) \cup \mathcal{L}_{n+1}^{n}$ and using (3.9) we obtain

$$
\begin{aligned}
\sum_{\mathbf{i} \in \mathcal{L}} \int_{X} \mathbb{P}_{x}\left(\mathbf{i}^{-1}\right) 1_{A}\left(S_{\mathbf{i}^{-1}}(x)\right) \mu_{*}(d x) & =\sum_{\mathbf{i} \in \mathcal{L}_{n+1}} \int_{X} \mathbb{P}_{x}\left(\mathbf{i}^{-1}\right) 1_{A}\left(S_{\mathbf{i}^{-1}}(x)\right) \mu_{*}(d x) \\
& +\sum_{\mathbf{i} \in \mathcal{L} \backslash \mathcal{L}_{n+1}} \int_{X} \mathbb{P}_{x}\left(\mathbf{i}^{-1}\right) 1_{A}\left(S_{\mathbf{i}^{-1}}(x)\right) \mu_{*}(d x) \\
& =\sum_{\mathbf{i} \in \mathcal{L}^{*}} \int_{X} \mathbb{P}_{x}\left(\mathbf{i}^{-1}\right) 1_{A}\left(S_{\mathbf{i}^{-1}}(x)\right) \mu_{*}(d x) .
\end{aligned}
$$


Clearly $\mathcal{L}^{*}$ is fundamental and $\mathcal{L}^{*} \subset \Omega_{\leq n}$. Now apply the induction hypothesis.

Let $L_{i}, i=1, \ldots, N$, be Lipschitz constants of $S_{i}$ and let $\Gamma_{0}$ be given by $(2.4)$. For $\mathbf{i}=\left(i_{1}, \ldots, i_{k}\right) \in \Omega_{*}$, we write

$$
L_{\mathbf{i}}=L_{i_{1}} \ldots L_{i_{k}} .
$$

For $\Gamma>\Gamma_{0}$ and $n_{0}, n \in \mathbb{N}, n \geq n_{0}$ we define

$$
Q_{n_{0}}^{n}(\Gamma)=\left\{\mathbf{i} \in \Omega_{\geq n}: L_{\mathbf{i} \mid k} \leq \Gamma^{k} \text { for } n_{0} \leq k \leq n\right\} .
$$

Lemma 3.4. Let $F$ be a bounded subset of $X$. Then for every $\Gamma \in\left(\Gamma_{0}, 1\right)$ and $n_{0} \in \mathbb{N}$ there exists $\alpha>0$ such that

$$
\mathbb{P}_{x}(\mathbf{i}) \geq \alpha \mathbb{P}_{y}(\mathbf{i}) \quad \text { for all } \mathbf{i} \in Q_{n_{0}}^{n}(\Gamma) \cap \Omega_{n}, n \geq n_{0} \text { and } x, y \in F .
$$

Proof. Fix $\Gamma \in\left(\Gamma_{0}, 1\right)$ and let $d=\operatorname{diam} F$. Let $\omega$ be as in (2.6), (2.7). Set

$$
\omega_{0}=\sum_{k=1}^{\infty} \omega\left(\Gamma^{k} d\right) .
$$

Clearly $\omega_{0}<\infty$. Fix $n_{0} \in \mathbb{N}$. Let $n \geq n_{0}$ and $x, y \in F$. For $\mathbf{i} \in Q_{n_{0}}^{n}(\Gamma) \cap \Omega_{n}$ we have

$$
\begin{aligned}
\mathbb{P}_{y}(\mathbf{i})= & p_{i_{1}}(y) p_{i_{2}}\left(S_{i_{1}}(y)\right) \ldots p_{i_{n}}\left(S_{i_{n-1}} \ldots S_{i_{1}}(y)\right) \\
= & \frac{p_{i_{1}}(y) \ldots p_{i_{n_{0}}}\left(S_{i_{n_{0}-1}} \circ \ldots \circ S_{i_{1}}(y)\right)}{p_{i_{1}}(x) \ldots p_{i_{0}}\left(S_{i_{n_{0}-1}} \circ \ldots \circ S_{i_{1}}(x)\right)} \\
& \times p_{i_{1}}(x) \ldots p_{i_{n_{0}}}\left(S_{i_{n_{0}-1}} \circ \ldots \circ S_{i_{1}}(x)\right) \\
& \times \prod_{k=n_{0}+1}^{n}\left[\left(1+\frac{p_{i_{k}}\left(S_{i_{k-1}} \circ \ldots \circ S_{i_{1}}(y)\right)-p_{i_{k}}\left(S_{i_{k-1}} \circ \ldots \circ S_{i_{1}}(x)\right)}{p_{i_{k}}\left(S_{i_{k-1}} \circ \ldots \circ S_{i_{1}}(x)\right)}\right)\right. \\
& \left.\quad \times p_{i_{k}}\left(S_{i_{k-1}} \circ \ldots \circ S_{i_{1}}(x)\right)\right] .
\end{aligned}
$$

Using the inequality $p_{i}(x) \geq \alpha_{0}$, conditions (2.6), (2.3) and definition (3.12) we obtain

$$
\begin{aligned}
\mathbb{P}_{y}(\mathbf{i}) \leq & \frac{\left(1-\alpha_{0}\right)^{n_{0}}}{\alpha_{0}^{n_{0}}} \\
& \times \prod_{k=n_{0}+1}^{n}\left(1+\frac{\omega\left(\varrho\left(S_{i_{k-1}} \circ \ldots \circ S_{i_{1}}(x), S_{i_{k-1}} \circ \ldots \circ S_{i_{1}}(y)\right)\right)}{\alpha_{0}}\right) \mathbb{P}_{x}(\mathbf{i}) \\
\leq & \left(\frac{1-\alpha_{0}}{\alpha_{0}}\right)^{n_{0}} \prod_{k=n_{0}+1}^{n}\left(1+\frac{\omega\left(\Gamma^{k-1} d\right)}{\alpha_{0}}\right) \mathbb{P}_{x}(\mathbf{i}) .
\end{aligned}
$$


Consequently,

$$
\mathbb{P}_{y}(\mathbf{i}) \leq\left(\frac{1-\alpha_{0}}{\alpha_{0}}\right)^{n_{0}} \prod_{k=n_{0}+1}^{\infty} e^{\omega\left(\Gamma^{k-1} d\right) / \alpha_{0}} \mathbb{P}_{x}(\mathbf{i})=\left(\frac{1-\alpha_{0}}{\alpha_{0}}\right)^{n_{0}} e^{\omega_{0} / \alpha_{0}} \mathbb{P}_{x}(\mathbf{i})
$$

Setting $\alpha=\alpha_{0}^{n_{0}}\left(1-\alpha_{0}\right)^{-n_{0}} e^{-\omega_{0} / \alpha_{0}}$ we complete the proof.

From now on assume that the constants $l_{i} \in(0,1), i=1, \ldots, N$, satisfy (2.3). Let $\gamma_{0}$ be given by (2.10). For $\gamma \in\left(0, \gamma_{0}\right)$ and $n \in \mathbb{N}$ define $J_{n}(\gamma)=\left\{i \in\{1, \ldots, N\}: l_{i} \leq \gamma^{n}\right\} \cup\left\{\mathbf{i} \in \Omega_{*}:|\mathbf{i}|>1\right.$ and $\left.l_{\mathbf{i}} \leq \gamma^{n}<l_{\mathbf{i}|\mathbf{i}|-1}\right\}$, where $l_{\mathbf{i}}$ is given by $(3.11)$.

Lemma 3.5. For every $\gamma \in\left(0, \gamma_{0}\right)$ and $n \in \mathbb{N}$ the set $J_{n}(\gamma)$ is fundamental for $(S, p)_{N}$.

Proof. Fix $\gamma \in\left(0, \gamma_{0}\right)$ and $n \in \mathbb{N}$. It is easy to verify that $J_{n}(\gamma) \subset \Omega_{\leq m}$, where $m$ is the least integer such that $\left(\max _{1 \leq i \leq N} l_{i}\right)^{m} \leq \gamma^{n}$. Consequently, $J_{n}(\gamma)$ is finite. Moreover, from the definition of $J_{n}(\gamma)$ it follows that if $\mathbf{i} \in$ $J_{n}(\gamma), \mathbf{j} \in \Omega_{*}$ and $\mathbf{j}>\mathbf{i}$, then $\mathbf{j} \notin J_{n}(\gamma)$. This implies that

$$
\Lambda(\mathbf{i}) \cap \Lambda(\mathbf{j})=\emptyset \quad \text { for } \mathbf{i}, \mathbf{j} \in J_{n}(\gamma), \mathbf{i} \neq \mathbf{j} .
$$

Finally, observe that for every $\mathbf{i} \in \Omega$ there is $k \in \mathbb{N}$ such that $\mathbf{i} \mid k \in J_{n}(\gamma)$. Consequently,

$$
\Omega=\bigcup_{\mathbf{i} \in J_{n}(\gamma)} \Lambda(\mathbf{i}) .
$$

By (3.13) and (3.14) for all $x \in X$ we have

$$
\sum_{\mathbf{i} \in J_{n}(\gamma)} \mathbb{P}_{x}(\mathbf{i})=\mathbb{P}_{x}\left(\bigcup_{\mathbf{i} \in J_{n}(\gamma)} \Lambda(\mathbf{i})\right)=\mathbb{P}_{x}(\Omega)=1 .
$$

Lemma 3.6. Assume that a family $S_{1}, \ldots, S_{N}$ satisfies the strong Moran condition and condition (2.3) with $l_{i} \in(0,1)$. Then for every $\gamma \in\left(0, \gamma_{0}\right)$, $n \in \mathbb{N}$ and $\mathbf{i}, \mathbf{j} \in J_{n}(\gamma), \mathbf{i} \neq \mathbf{j}$, we have

$$
\operatorname{dist}\left(S_{\mathbf{i}^{-1}}(F), S_{\mathbf{j}^{-1}}(F)\right) \geq \gamma^{n} \sigma,
$$

where the set $F$ and the constant $\sigma$ satisfy (2.11), (2.12).

Proof. Fix $\gamma \in\left(0, \gamma_{0}\right), n \in \mathbb{N}$ and $\mathbf{i}, \mathbf{j} \in J_{n}(\gamma), \mathbf{i} \neq \mathbf{j}$. Suppose $\mathbf{i}=$ $\left(i_{1}, \ldots, i_{p}\right)$ and $\mathbf{j}=\left(j_{1}, \ldots, j_{q}\right)$. Since $J_{n}(\gamma)$ is fundamental, there exists an integer $m \leq \min \{p, q\}$ such that $i_{m} \neq j_{m}$, but $i_{k}=j_{k}$ for $k<m$. From the strong Moran condition it follows immediately that $S_{\mathbf{i}^{-1}}(F) \subset$ $S_{i_{1}} \circ \ldots \circ S_{i_{m}}(F), S_{\mathbf{j}^{-1}}(F) \subset S_{j_{1}} \circ \ldots \circ S_{j_{m}}(F)$ and $\operatorname{dist}\left(S_{i_{m}}(F), S_{j_{m}}(F)\right) \geq \sigma$. Consequently,

$$
\begin{aligned}
\operatorname{dist}\left(S_{\mathbf{i}^{-1}}(F), S_{\mathbf{j}^{-1}}(F)\right) & \geq \operatorname{dist}\left(S_{i_{1}} \circ \ldots \circ S_{i_{m}}(F), S_{j_{1}} \circ \ldots \circ S_{j_{m}}(F)\right) \\
& \geq l_{i_{1}} \ldots l_{i_{m}-1} \sigma \geq \gamma^{n} \sigma .
\end{aligned}
$$


LEMma 3.7. Assume that an IFS $\left(S, p_{N}\right)$ satisfies $(2.3)$ with $l_{i} \in(0,1)$, $i=1, \ldots, N$. Let $\gamma_{0}$ be given by (2.10). Then for every $\gamma \in\left(0, \gamma_{0}\right)$ there is $\beta>0$ such that

$$
\sum_{\mathbf{i} \in J_{n}^{*}(\gamma)} \mathbb{P}_{x}(\mathbf{i}) \geq \beta \quad \text { for every } x \in X,
$$

where

$$
J_{n}^{*}(\gamma)=\left\{\mathbf{i} \in J_{n}(\gamma): \mathbf{i}^{-1} \in J_{n}(\gamma)\right\}
$$

Proof. Without any loss of generality we can assume that

$$
l_{1} \leq l_{i} \leq l_{N} \quad \text { for } i=1, \ldots, N .
$$

Observe that if $\mathbf{i}=\left(i_{1}, \ldots, i_{k}\right) \in J_{n}(\gamma)$ and $i_{k}=N$ then $\mathbf{i}^{-1} \in J_{n}(\gamma)$. Moreover, for every $\mathbf{i}=\left(i_{1}, \ldots, i_{k}\right) \in J_{n}(\gamma)$ there exists a unique element

$$
\tau(\mathbf{i})=\left(i_{1}, \ldots, i_{k-1}, N, \ldots, N\right)
$$

which belongs to $J_{n}(\gamma)$. In this way formula (3.17) defines a one-to-one map from $J_{n}(\gamma)$ into $J_{n}(\gamma)$. Note also that

$$
(\tau(\mathbf{i}))^{-1} \in J_{n}(\gamma) \quad \text { for every } \mathbf{i} \in J_{n}(\gamma) .
$$

Fix $\mathbf{i}=\left(i_{1}, \ldots, i_{k}\right) \in J_{n}(\gamma)$ and let $m_{0}$ be such that $l_{N}^{m_{0}} \leq l_{1}$. Since $l_{\mathbf{i}} \leq \gamma^{n}$ and $l_{1} \leq l_{i}$ for $i=1, \ldots, N$, we have

$$
|\tau(\mathbf{i})| \leq|\mathbf{i}|-1+m_{0},
$$

which means that in (3.17) the number $N$ appears at most $m_{0}$ times. Now it is easy to see that for every $\mathbf{i} \in J_{n}(\gamma)$,

$$
\operatorname{card}\left\{\mathbf{j} \in J_{n}(\gamma): \tau(\mathbf{j})=\tau(\mathbf{i})\right\} \leq N^{m_{0}}
$$

(card stands for cardinality). By (3.17), (2.15) and (2.17) for every $x \in X$ we have

$$
\begin{aligned}
\mathbb{P}_{x}(\tau(\mathbf{i})) & =\mathbb{P}_{x}\left(i_{1}, \ldots, i_{k-1}, N, \ldots, N\right) \\
& \geq \mathbb{P}_{x}\left(i_{1}, \ldots, i_{k-1}\right) \alpha_{0}^{m_{0}} \geq \mathbb{P}_{x}(\mathbf{i}) \alpha_{0}^{m_{0}},
\end{aligned}
$$

where $\alpha_{0}$ is given by (2.5). By Lemma 3.5 and (3.5), (3.20) and (3.19) we have

$$
1=\sum_{\mathbf{i} \in J_{n}(\gamma)} \mathbb{P}_{x}(\mathbf{i}) \leq \alpha_{0}^{-m_{0}} \sum_{\mathbf{i} \in J_{n}(\gamma)} \mathbb{P}_{x}(\tau(\mathbf{i})) \leq N^{m_{0}} \alpha_{0}^{-m_{0}} \sum_{\mathbf{i} \in J_{n}^{*}(\gamma)} \mathbb{P}_{x}(\mathbf{i}) .
$$

Setting $\beta=\left(\alpha_{0} / N\right)^{m_{0}}$ completes the proof. 


\section{Upper estimate of capacity}

THEOREM 4.1. Assume that an IFS $(S, p)_{N}$ satisfies (2.3)-(2.7) and let $\mu_{*} \in \mathcal{M}_{1}$ be the corresponding invariant measure. Then

$$
\overline{\operatorname{Cap}}_{L}\left(\mu_{*}\right) \leq \frac{\log \delta_{0}}{\log \Gamma_{0}}
$$

where $\Gamma_{0}$ and $\delta_{0}$ are given by (2.4) and (2.9), respectively.

Proof. Fix $\eta>0$. Let $\mu_{*}$ be the unique invariant probability measure for the IFS $(S, p)_{N}$ and let $K$ be a compact subset of $X$ such that $\mu_{*}(K) \geq$ $1-\eta / 4$. Set $d=\operatorname{diam} K$. Fix $x_{0} \in K$. By Lemma 3.1 (with $L_{i}$ and $p_{i}(x)$ in place of $f_{i}(x)$ and $\Gamma_{0}$ and $\delta_{0}$ in place of $\Delta$ and $\delta$, respectively) there exists a measurable set $\Omega_{0} \subset \Omega$ with $\mathbb{P}_{x_{0}}\left(\Omega_{0}\right)=1$ such that, for all $\mathbf{i} \in \Omega_{0}$,

$$
\begin{aligned}
\limsup _{n \rightarrow \infty} \frac{1}{n} \log \left(L_{\mathbf{i} \mid n}\right) & \leq \log \Gamma_{0}, \\
\liminf _{n \rightarrow \infty} \frac{1}{n} \log \left(\mathbb{P}_{x_{0}}(\mathbf{i} \mid n)\right) & \geq \log \delta_{0},
\end{aligned}
$$

where $L_{\mathbf{i} \mid n}=L_{i_{1}} \ldots L_{i_{n}}$. By Lemma 3 of [8] the measure $\mathbb{P}_{x}, x \in X$, is absolutely continuous with respect to $\mathbb{P}_{x_{0}}$. Thus $\mathbb{P}_{x}\left(\Omega_{0}\right)=1$ for $x \in X$.

Choose $\Gamma \in\left(\Gamma_{0}, 1\right), \delta \in\left(0, \delta_{0}\right)$ and define the sequence $\left(\Omega_{0}(n)\right)_{n \geq 1}$ of measurable subsets of $\Omega_{0}$ by

$$
\Omega_{0}(n)=\left\{\mathbf{i} \in \Omega_{0}: \mathbb{P}_{x_{0}}(\mathbf{i} \mid k) \geq \delta^{k} \text { and } L_{\mathbf{i} \mid k} \leq \Gamma^{k} \text { for } k \geq n\right\} .
$$

Obviously $\Omega_{0}(n) \subset \Omega_{0}(n+1)$ for $n \in \mathbb{N}$. Moreover, from (4.2), (4.3) and the choice of $\Gamma$ and $\delta$ it follows that $\Omega_{0}=\bigcup_{n=1}^{\infty} \Omega_{0}(n)$. Consequently,

$$
\lim _{n \rightarrow \infty} \mathbb{P}_{x}\left(\Omega_{0}(n)\right)=1 \quad \text { for } x \in X .
$$

By Lemma 4.1 of [24] the function $x \mapsto \mathbb{P}_{x}\left(\Omega_{0}(n)\right)$ is Borel measurable for each $n \in \mathbb{N}$. By $(4.4)$ the sequence $\left(\mathbb{P}_{x}\left(\Omega_{0}(n)\right)\right)_{n>1}$ is a.s. convergent and so convergent with respect to the measure $\mu_{*}$. Hence there exists $n_{0} \in \mathbb{N}$ such that

$$
\mu_{*}\left(\left\{x \in K: \mathbb{P}_{x}\left(\Omega_{0}(n)\right)>1-\eta / 2\right\}\right) \geq 1-\eta / 2 \quad \text { for } n \geq n_{0} .
$$

By the invariance of $\mu_{*}$, for all $n \in \mathbb{N}$ and $A \in \mathcal{B}(X)$ we have

$$
\mu_{*}(A)=P^{n} \mu_{*}(A)=\sum_{\mathbf{i} \in \Omega_{n}} \int_{X} \mathbb{P}_{x}(\mathbf{i}) 1_{A}\left(S_{\mathbf{i}}(x)\right) \mu_{*}(d x) .
$$

Now, for $n \in \mathbb{N}$ define

$$
D_{n}=\bigcup_{\mathbf{i} \in \Omega_{0}^{n}(n)} B\left(S_{\mathbf{i}}\left(x_{0}\right), \varepsilon_{n}\right),
$$

where

$$
\Omega_{0}^{n}(n)=\left\{\mathbf{i} \mid n: \mathbf{i} \in \Omega_{0}(n)\right\} \quad \text { and } \quad \varepsilon_{n}=\Gamma^{n} \operatorname{diam} K .
$$


Observe that

$$
\varrho\left(S_{\mathbf{i}}(x), S_{\mathbf{i}}\left(x_{0}\right)\right) \leq \varepsilon_{n} \quad \text { for } \mathbf{i} \in \Omega_{0}^{n}(n) \text { and } x \in K .
$$

Using (4.5)-(4.7) and the inclusion $\left.\Omega_{0}(n) \subset \Lambda\left(\Omega_{0}^{n}(n)\right)\right)$, for every $n \geq n_{0}$ we have

$$
\begin{aligned}
\mu_{*}\left(D_{n}\right) & =\sum_{\mathbf{i} \in \Omega_{n}} \int_{X} \mathbb{P}_{x}(\mathbf{i}) 1_{D_{n}}\left(S_{\mathbf{i}}(x)\right) \mu_{*}(d x) \\
& \geq \sum_{\mathbf{i} \in \Omega_{0}^{n}(n)} \int_{X} \mathbb{P}_{x}(\mathbf{i}) 1_{D_{n}}\left(S_{\mathbf{i}}(x)\right) \mu_{*}(d x) \\
& \geq \int_{K} \sum_{\mathbf{i} \in \Omega_{0}^{n}(n)} \mathbb{P}_{x}(\mathbf{i}) \mu_{*}(d x)=\int_{K} \mathbb{P}_{x}\left(\Omega_{0}^{n}(n)\right) \mu_{*}(d x) \\
& \geq \int_{K} \mathbb{P}_{x}\left(\Omega_{0}(n)\right) \mu_{*}(d x) \geq(1-\eta / 2)(1-\eta / 2)>1-\eta .
\end{aligned}
$$

From this inequality and the definition of $D_{n}$ it follows that $N\left(\varepsilon_{n}, \eta\right) \leq N_{0}$, where $N_{0}=$ card $\Omega_{0}^{n}(n)$ and $N\left(\varepsilon_{n}, \eta\right)$ comes from the definition of capacity. Since $\mathbb{P}_{x_{0}}(\mathbf{i}) \geq \delta^{n}$ for $\mathbf{i} \in \Omega_{0}^{n}(n)$ and $\sum_{\mathbf{i} \in \Omega_{0}^{n}(n)} \mathbb{P}_{x_{0}}(\mathbf{i}) \leq 1$, we have $N_{0} \delta^{n} \leq 1$. Consequently, $N\left(\varepsilon_{n}, \eta\right) \leq \delta^{-n}$. By Remark 2.1 we now have

$$
\begin{aligned}
\limsup _{\varepsilon \rightarrow 0} \frac{\log N(\varepsilon, \eta)}{-\log \varepsilon} & =\limsup _{n \rightarrow \infty} \frac{\log N\left(\varepsilon_{n}, \eta\right)}{-\log \varepsilon_{n}} \\
& \leq \limsup _{n \rightarrow \infty} \frac{\log \delta^{-n}}{-\log \left(\Gamma^{n} \operatorname{diam} K\right)}=\frac{\log \delta}{\log \Gamma} .
\end{aligned}
$$

Letting $\delta \rightarrow \delta_{0}$ and $\Gamma \rightarrow \Gamma_{0}$ we conclude that

$$
\limsup _{\varepsilon \rightarrow 0} \frac{\log N(\varepsilon, \eta)}{-\log \varepsilon} \leq \frac{\log \delta_{0}}{\log \Gamma_{0}}
$$

Since $\eta>0$ was arbitrary, the proof is complete.

\section{Lower estimate of capacity}

TheOREm 5.1. Assume that an IFS $(S, p)_{N}$ satisfies (2.3)-(2.7) and $\mu_{*} \in \mathcal{M}_{1}$ is the corresponding unique invariant measure. Moreover, assume that the functions $S_{1}, \ldots, S_{N}$ satisfy the strong Moran condition. Then

$$
\operatorname{Cap}_{L}\left(\mu_{*}\right) \geq \frac{\log \Delta_{0}}{\log \gamma_{0}}
$$

where $\Delta_{0}$ and $\gamma_{0}$ are given by (2.8) and (2.9), respectively.

Proof. Consider first the case $l_{i}<1, i=1, \ldots, N$, where the $l_{i}$ satisfy (2.3). Let $F$ be a closed set satisfying (2.11) and (2.12). Since $F$ is invariant for $S_{1}, \ldots, S_{N}$, we have supp $\mu_{*} \subset F$. Choose $x_{0} \in F$. By Lemma 3.1 
(with $p_{i}$ and $\Delta_{0}$ or $L_{i}$ and $\Gamma_{0}$ in place of $f_{i}$ and $\Delta$, and $l_{i}$ and $\gamma$ in place of $f_{i}$ and $\gamma_{0}$, respectively) we have

$$
\begin{aligned}
& \limsup _{n \rightarrow \infty} \frac{1}{n} \log \left(\mathbb{P}_{x_{0}}(\mathbf{i} \mid n)\right) \leq \log \Delta_{0} \quad \mathbb{P}_{x_{0}} \text {-a.s. }, \\
& \limsup _{n \rightarrow \infty} \frac{1}{n} \log \left(L_{\mathbf{i} \mid n}\right) \leq \log \Gamma_{0} \quad \mathbb{P}_{x_{0}} \text {-a.s. }, \\
& \liminf _{n \rightarrow \infty} \frac{1}{n} \log \left(l_{\mathbf{i} \mid n}\right) \geq \log \gamma_{0} \quad \mathbb{P}_{x_{0}} \text {-a.s. }
\end{aligned}
$$

Fix $\gamma \in\left(0, \gamma_{0}\right), \Gamma \in\left(\Gamma_{0}, 1\right)$ and $\Delta \in\left(\Delta_{0}, 1\right)$. Let $n_{0} \in \mathbb{N}$ and let $\alpha$ be as in Lemma 3.4. By (5.2)-(5.4) there exists $n_{1} \geq n_{0}$ such that

$$
\begin{array}{r}
\mathbb{P}_{x_{0}}\left(\left\{\mathbf{i} \in \Omega: \mathbb{P}_{x_{0}}(\mathbf{i} \mid n) \leq \Delta^{n} \text { for } n \geq n_{1}\right\}\right) \geq 1-\beta / 6, \\
\mathbb{P}_{x_{0}}\left(\left\{\mathbf{i} \in \Omega: L_{\mathbf{i} \mid n} \leq \Gamma^{n} \text { for } n \geq n_{1}\right\}\right) \geq 1-\beta / 6, \\
\mathbb{P}_{x_{0}}\left(\left\{\mathbf{i} \in \Omega: l_{\mathbf{i} \mid n} \geq \gamma^{n} \text { for } n \geq n_{1}\right\}\right) \geq 1-\beta / 6,
\end{array}
$$

where $\beta$ is as in Lemma 3.7. Now choose $n_{*} \in \mathbb{N}$ such that

$$
\min \left\{|\mathbf{i}|: \mathbf{i} \in J_{n}(\gamma)\right\} \geq n_{1} \quad \text { for } n \geq n_{*} .
$$

For $n \geq n_{*}$ define

$$
\begin{aligned}
& J_{n}^{0}(\gamma)=\left\{\mathbf{i} \in J_{n}(\gamma): \mathbb{P}_{x_{0}}\left(\mathbf{i}^{-1} \mid k\right) \leq \Delta^{k}, L_{\mathbf{i}^{-1} \mid k} \leq \Gamma^{k}\right. \\
& \text { and } \left.l_{\mathbf{i}^{-1} \mid k} \geq \gamma^{k} \text { for } k \in \mathbb{N}, n_{1} \leq k \leq|\mathbf{i}|\right\} .
\end{aligned}
$$

By (5.5)-(5.8) and Lemma 3.7 we have

$$
\sum_{\mathbf{i} \in J_{n}^{0}(\gamma)} \mathbb{P}_{x_{0}}\left(\mathbf{i}^{-1}\right) \geq \beta / 2 \quad \text { for } n \geq n_{1} .
$$

Since $\mathbf{i}^{-1} \in Q_{n_{1}}^{n}(\gamma)$ for $\mathbf{i} \in J_{n}^{0}(\gamma)$, Lemma 3.4 yields

$$
\alpha^{-1} \mathbb{P}_{x_{0}}\left(\mathbf{i}^{-1}\right) \geq \mathbb{P}_{x}\left(\mathbf{i}^{-1}\right) \geq \alpha \mathbb{P}_{x_{0}}\left(\mathbf{i}^{-1}\right) \quad \text { for } \mathbf{i} \in J_{n}^{0}(\gamma) \text { and } x \in F .
$$

From (5.9) and (5.10) it follows that

$$
\begin{aligned}
\sum_{\mathbf{i} \in J_{n}^{0}(\gamma)} \mathbb{P}_{x}\left(\mathbf{i}^{-1}\right) \geq \alpha \beta / 2, \\
\mathbb{P}_{x}\left(\mathbf{i}^{-1}\right) \leq \alpha^{-1} \Delta^{|\mathbf{i}|},
\end{aligned}
$$

for all $n \geq n_{*}, x \in F$ and $\mathbf{i} \in J_{n}^{0}(\gamma)$. Since for $\mathbf{i} \in J_{n}^{0}(\gamma)$ we have $l_{\mathbf{i}} \leq \gamma^{n}$ and $l_{\mathbf{i}^{-1} \mid k} \geq \gamma^{k}$ for $n_{1} \leq k \leq|\mathbf{i}|$, it follows that $|\mathbf{i}| \geq n$. Hence

$$
\mathbb{P}_{x}\left(\mathbf{i}^{-1}\right) \leq \alpha^{-1} \Delta^{n} \quad \text { for } \mathbf{i} \in J_{n}^{0}(\gamma) .
$$

For $n \geq n_{*}$ define

$$
D_{n}=\bigcup_{\mathbf{i} \in J_{n}^{0}(\gamma)} S_{\mathbf{i}^{-1}}(F)
$$


Since by Lemma 3.5 the set $J_{n}(\gamma)$ is fundamental, Lemma 3.3 and (5.11) show that

$$
\begin{aligned}
\mu_{*}\left(D_{n}\right) & =\sum_{\mathbf{i} \in J_{n}(\gamma)} \int_{X} \mathbb{P}_{x}\left(\mathbf{i}^{-1}\right) 1_{D_{n}}\left(S_{\mathbf{i}^{-1}}(x)\right) \mu_{*}(d x) \\
& \geq \int_{F} \sum_{\mathbf{i} \in J_{n}^{0}(\gamma)} \mathbb{P}_{x}\left(\mathbf{i}^{-1}\right) 1_{D_{n}}\left(S_{\mathbf{i}^{-1}}(x)\right) \mu_{*}(d x) \\
& =\int_{F} \sum_{\mathbf{i} \in J_{n}^{0}(\gamma)} \mathbb{P}_{x}\left(\mathbf{i}^{-1}\right) \mu_{*}(d x) \geq \alpha \beta / 2
\end{aligned}
$$

for $n \geq n_{*}$.

By Lemmas 3.3, 3.5 and 3.6, the inclusion supp $\mu_{*} \subset F$ and inequality (5.13), for every $\mathbf{j} \in J_{n}^{0}(\gamma)$ and $n \geq n_{*}$, we have

$$
\begin{aligned}
\mu_{*}\left(S_{\mathbf{j}^{-1}}(F)\right) & =\sum_{\mathbf{i} \in J_{n}(\gamma)} \int_{X} \mathbb{P}_{x}\left(\mathbf{i}^{-1}\right) 1_{S_{\mathbf{j}^{-1}}(F)}\left(S_{\mathbf{i}^{-1}}(x)\right) \mu_{*}(d x) \\
& =\sum_{\mathbf{i} \in J_{n}^{0}(\gamma)} \int_{F} \mathbb{P}_{x}\left(\mathbf{i}^{-1}\right) 1_{S_{\mathbf{j}^{-1}}(F)}\left(S_{\mathbf{i}^{-1}}(x)\right) \mu_{*}(d x) \\
& =\int_{F} \mathbb{P}_{x}\left(\mathbf{j}^{-1}\right) \mu_{*}(d x) \leq \Delta^{n} / \alpha,
\end{aligned}
$$

since $S_{\mathbf{i}^{-1}}(F) \cap S_{\mathbf{j}^{-1}}(F)=\emptyset$ for $\mathbf{i} \neq \mathbf{j}, \mathbf{i}, \mathbf{j} \in J_{n}^{0}(\gamma)$. Define $\varepsilon_{n}=\gamma^{n} \sigma / 2$ for $n \geq n_{*}$, where $\sigma>0$ is given by (2.12). By (3.15) every ball $B$ with radius $\varepsilon_{n}$ meets at most one set $S_{\mathbf{i}^{-1}}(F)$ for $\mathbf{i} \in J_{n}^{0}(\gamma)$. The inclusion supp $\mu_{*} \subset$ $\bigcup_{\mathbf{i} \in J_{n}(r)} S_{\mathbf{i}^{-1}}(F)$ then implies that to cover a set of $\mu_{*}$-measure greater than or equal to $1-\eta$ (with $\eta \leq \alpha \beta / 2$ ) we need at least $\alpha(1-\eta) \Delta^{-n}$ balls with radius $\varepsilon_{n}$. Thus $N\left(\varepsilon_{n}, \eta\right) \geq \alpha(1-\eta) \Delta^{-n}$ for $n \geq n_{*}$. Consequently,

$$
\liminf _{\varepsilon \rightarrow 0} \frac{\log N(\varepsilon, \eta)}{-\log \varepsilon} \geq \liminf _{n \rightarrow \infty} \frac{\log \left(\alpha(1-\eta) \Delta^{-n}\right)}{-\log \varepsilon_{n}}=\frac{\log \Delta}{\log \gamma} .
$$

Thus

$$
\operatorname{Cap}_{L}\left(\mu_{*}\right) \geq \frac{\log \Delta}{\log \gamma}
$$

and letting $\Delta \rightarrow \Delta_{0}$ and $\gamma \rightarrow \gamma_{0}$ we conclude that

$$
\operatorname{Cap}_{L}\left(\mu_{*}\right) \geq \frac{\log \Delta_{0}}{\log \gamma_{0}} .
$$

Suppose now that some of the $l_{i}$ 's are equal to 1 . Choose $\bar{l}_{i}<l_{i}, i=$ $1, \ldots, N$. Since

$$
\sup _{x \in X} \prod_{i=1}^{N} \bar{l}_{i}^{p_{i}(x)} \rightarrow \sup _{x \in X} \prod_{i=1}^{N} l_{i}^{p_{i}(x)}
$$

as $\bar{l}_{i} \rightarrow l_{i}$ for $i \in\{1, \ldots, N\}$, the statement of Theorem 5.1 follows. 
Acknowledgements. Tomasz Szarek was supported by Foundation for Polish Science and the State Committee for Scientific Research (Poland), Grant No. 2 P03A 01016.

\section{References}

[1] M. Arbeiter and N. Patzschke, Random self-similar multifractals, Math. Nachr. 181 (1996), 5-42.

[2] M. F. Barnsley, S. G. Demko, J. H. Elton and J. S. Geronimo, Invariant measures arising from iterated function systems with place dependent probabilities, Ann. Inst. H. Poincaré 24 (1988), 367-394.

[3] M. Barnsley, J. Elton and D. Hardin, Recurrent iterated function systems, Constr. Approx. 5 (1989), 3-31.

[4] T. Bedford, Dimension and dynamics for fractal recurrent sets, J. London Math. Soc. 33 (1986), 98-100.

[5] C. D. Cutler, Connecting ergodicity and dimension in dynamical systems, Ergodic Theory Dynam. Systems 10 (1990), 451-462.

[6] P. Diaconis and D. Freedman, Iterated random functions, SIAM Rev. 41 (1999), 45-76.

[7] R. M. Dudley, Probabilities and Metrics, Aarhus Univ., Aarhus, 1976.

[8] J. H. Elton, An ergodic theorem for iterated maps, Ergodic Theory Dynam. Systems 7 (1987), 481-488.

[9] S. Ethier and T. Kurtz, Markov Processes, Wiley, New York, 1986.

[10] W. Feller, An Introduction to Probability Theory and its Applications, Wiley, New York, 1981.

[11] R. Fortet et B. Mourier, Convergence de la répartition empirique vers la répartition théorétique, Ann. Sci. École Norm. Sup. 70 (1953), 267-285.

[12] J. S. Geronimo and D. P. Hardin, An exact formula for the measure dimensions associated with a class of piecewise linear maps, Constr. Approx. 5 (1989), 89-98.

[13] J. E. Hutchinson, Fractals and self-similarity, Indiana Univ. Math. J. 30 (1981), 713-743.

[14] A. N. Kolmogorov, A new invariant for transitive dynamical systems, Dokl. Akad. Nauk SSSR 119 (1958), 861-864 (in Russian).

[15] A. Lasota and J. Myjak, Semifractals on Polish spaces, Bull. Polish Acad. Sci. Math. 46 (1998), 179-196.

[16] A. Lasota and J. A. Yorke, Lower bound technique for Markov operators and iterated function systems, Random Comput. Dynam. 2 (1994), 41-77.

[17] F. Ledrappier, Some relations between dimension and Lyapunov exponents, Comm. Math. Phys. 81 (1981), 95-102.

[18] R. D. Mauldin and S. C. Williams, Hausdorff dimension in graph directed constructions, Trans. Amer. Math. Soc. 309 (1988), 811-829.

[19] P. Moran, Additive functions of intervals and Hausdorff dimension, Proc. Cambridge Philos. Soc. 42 (1946), 15-23.

[20] J. Myjak and T. Szarek, On Hausdorff dimension of invariant measures arising from iterated function systems, Ann. Mat. Pura Appl., to appear.

[21] L. Olsen, A multifractal formalism, Adv. Math. 116 (1995), 82-195.

[22] Ya. B. Pesin, On rigorous mathematical definitions of correlation dimension and generalized spectrum for dimensions, J. Statist. Phys. 71 (1993), 529-547. 
[23] T. Szarek, Markov operators acting on Polish spaces, Ann. Polon. Math. 67 (1997), 247-257.

[24] - The dimension of self-similar measures, Bull. Polish Acad. Sci. Math. 48 (2000), 293-302.

[25] L. S. Young, Dimension, entropy and Lyapunov exponents, Ergodic Theory Dynam. Systems 2 (1982), 109-124.

Dipartamento di Matematica Pura ed Applicata

Università di L'Aquila

via Vetoio

67100 L'Aquila, Italy

E-mail: myjak@univag.it

WMS AGH

Al. Mickiewicza 30

30-059 Kraków, Poland
Institute of Mathematics Polish Academy of Sciences Bankowa 14 40-007 Katowice, Poland E-mail: szarek@ux2.math.us.edu.pl

Department of Mathematics Technical University of Rzeszów W. Pola 6 35-959 Rzeszów, Poland

Reçu par la Rédaction le 15.11.2000

Révisé le 19.3.2001 et 5.7.2001 